\title{
Research Article \\ Shift Unitary Transform for Constructing Two-Dimensional Wavelet Filters
}

\author{
Fei $\mathrm{Li}^{1}$ and Jianwei Yang ${ }^{2}$ \\ ${ }^{1}$ School of Economics, Beijing Technology and Business University, Beijing 100048, China \\ ${ }^{2}$ College of Math and Physics, Nanjing University of Information Science and Technology, \\ Nanjing 210044, China \\ Correspondence should be addressed to Jianwei Yang, yjianw2002@yahoo.com.cn
}

Received 13 March 2011; Revised 27 May 2011; Accepted 8 June 2011

Academic Editor: F. Marcellán

Copyright (C) 2011 F. Li and J. Yang. This is an open access article distributed under the Creative Commons Attribution License, which permits unrestricted use, distribution, and reproduction in any medium, provided the original work is properly cited.

\begin{abstract}
Due to the difficulty for constructing two-dimensional wavelet filters, the commonly used wavelet filters are tensor-product of one-dimensional wavelet filters. In some applications, more perfect reconstruction filters should be provided. In this paper, we introduce a transformation which is referred to as Shift Unitary Transform (SUT) of Conjugate Quadrature Filter (CQF). In terms of this transformation, we propose a parametrization method for constructing two-dimensional orthogonal wavelet filters. It is proved that tensor-product wavelet filters are only special cases of this parametrization method. To show this, we introduce the SUT of one-dimensional CQF and present a complete parametrization of one-dimensional wavelet system. As a result, more ways are provided to randomly generate two-dimensional perfect reconstruction filters.
\end{abstract}

\section{Introduction}

In her celebrated paper [1], Daubechies constructed a family of compactly supported orthonormal scaling functions and their corresponding orthonormal wavelets. Since then, wavelets with compact support have been found to be very useful in applications (see [2] and references therein). By now, the theory for the construction of one-dimensional wavelets is well developed [1, 3-9]. But, there still exists many open problems for the construction of multidimensional wavelets ([10-13], etc.).

To apply wavelet methods to digital image processing, two-dimensional wavelets have to be constructed. The most common wavelets used for image processing are tensorproduct of one-dimensional wavelets (separable wavelets). Nevertheless, separable wavelets have a number of drawbacks [11]. Nonseparable wavelets offer the hope of more isotropic analysis ([14-16], etc.). Many efforts have been made on constructing nonseparable wavelets. However, up to now, only a few constructions have been published. Cohen and Daubechies 
[14] used the univariate construction [1] to produce nonseparable scaling function with higher accuracy. Continuous nonseparable scaling functions were constructed by He and Lai [12] and Kovačević [15]. Arbitrarily smooth nonseparable orthogonal wavelets were constructed by Ayache [10] and Belogay and wang [11]. Recently, Lai [13] proposed a constructive method to find compactly supported orthonormal wavelets for any given compactly supported scaling function in the multivariate setting.

In some applications of wavelets, such as wavelet-based watermarking $[17,18]$, parametrization of two-dimensional wavelet filters is preferred. To make some wavelet-based watermarking schemes more robust, we need to create as many ways as possible to randomly generate perfect reconstruction filters [17]. The ample choices of wavelet filters will increase the difficulty for counterfeiters to gain the exact knowledge of the filters (see [17-20]). But in methods available, to derive two-dimensional wavelet filters, one has to solve transcendental constraints for the parameters. Hence, wavelet filters used in wavelet-based watermarking schemes, such as [17-20], are only tensor-product of one-dimensional wavelets.

In this paper, a transformation that we refer to as Shift Unitary Transform (SUT) of Conjugate Quadrature Filter (CQF) is proposed. In terms of this transformation, we present a parametrization method for constructing two-dimensional orthogonal wavelet filters. The choosing of the parameters is not restricted by any implicit condition. It is proved that tensorproduct wavelet filters are only special cases of this parametrization method. Therefore, more ways are provided to randomly generate perfect reconstruction filters. The ample choices of wavelet filters will increase the difficulty for counterfeiters to gain the exact knowledge of the filters and make watermarking schemes based on wavelet filters more robust [17-20].

First of all, it should be pointed out that all the filters along the paper are FIR (finite impulse response). For a matrix $A$, we denote its transpose by $A^{T}$ in this paper.

To show that tensor-product wavelet filters are only special cases of our construction, we introduce the SUT of one-dimensional CQF and present a complete parametrization of one-dimensional wavelet system in Section 2. In Section 3, we show that tensor-product orthogonal wavelet filters can be constructed by SUT of CQF. Then, based on SUT of twodimensional CQF, a parameterized method is presented for constructing real-valued two-dimensional orthogonal wavelet filters. Finally, nonseparable wavelet filters are derived. Conclusion remarks are given in Section 4.

\section{Parametrization of One-Dimensional Wavelet Filters}

To construct one-dimensional orthogonal scaling function

$$
\varphi(x)=\sqrt{2} \sum_{k \in \mathbb{Z}} h_{k} \varphi(2 x-k)
$$

we need construct sequences $\left\{h_{k}\right\}_{k \in \mathbb{Z}}$ such that (see $[4,21]$ and many others)

$$
\begin{gathered}
\sum_{k \in \mathbb{Z}} h_{k}=\sqrt{2}, \\
\sum_{k \in \mathbb{Z}} h_{2 k}=\sum_{k \in \mathbb{Z}} h_{2 k+1}, \\
\sum_{k \in \mathbb{Z}} h_{k} h_{k+2 \alpha}=\delta_{0, \alpha}, \quad \alpha \in \mathbb{Z},
\end{gathered}
$$


where $\delta$ denotes the Kronecker delta

$$
\delta_{\alpha, \beta}=\left\{\begin{array}{ll}
0, & \alpha \neq \beta, \\
1, & \alpha=\beta .
\end{array} \quad \alpha, \beta \in \mathbb{Z}\right.
$$

The sequence $\left\{h_{k}\right\}_{k \in \mathbb{Z}}$ which satisfies (2.4) is called a one-dimensional CQF, and the sequence $\left\{h_{k}\right\}_{k \in \mathbb{Z}}$, which satisfies (2.2), (2.3), (2.4) simultaneously, is called one-dimensional orthogonal low-pass wavelet filter.

Definition 2.1. Let $\left\{h_{n}\right\}_{n \in \mathbb{Z}}$ be a one-dimensional CQF and $G$ an arbitrary $2 \times 2$ orthogonal matrix. $\left\{\tilde{h}_{n}\right\}_{n \in \mathbb{Z}}$ is called the shift unitary transform (SUT) of $\left\{h_{n}\right\}_{n \in \mathbb{Z}}$ by $G$ if $\left\{\tilde{h}_{n}\right\}_{n \in \mathbb{Z}}$ satisfies

$$
\tilde{\Upsilon}_{i}=\Lambda_{i} G, \quad i \in \mathbb{Z},
$$

and $\left\{\widetilde{h_{n}^{\prime}}\right\}_{n \in \mathbb{Z}}$ is called the inverse shift unitary transform (ISUT) of $\left\{h_{n}\right\}_{n \in \mathbb{Z}}$ by $G$ if $\left\{\widetilde{h_{n}^{\prime}}\right\}_{n \in \mathbb{Z}}$ satisfies

$$
\widetilde{\Lambda_{i}^{\prime}}=\Upsilon_{i} G, \quad i \in \mathbb{Z},
$$

where for any $i \in Z$,

$$
\tilde{\Upsilon}_{i}=\left(\begin{array}{c}
\tilde{h}_{2 i} \\
\tilde{h}_{2 i+1}
\end{array}\right)^{T}, \quad \Lambda_{i}=\left(\begin{array}{c}
h_{2 i} \\
h_{2 i-1}
\end{array}\right)^{T}, \quad \widetilde{\Lambda_{i}^{\prime}}=\left(\begin{array}{c}
\widetilde{h_{2 i}^{\prime}} \\
\widetilde{h_{2 i-1}^{\prime}}
\end{array}\right)^{T}, \quad \Upsilon_{i}=\left(\begin{array}{c}
h_{2 i} \\
h_{2 i+1}
\end{array}\right)^{T} .
$$

The SUT and the ISUT of $\left\{h_{n}\right\}_{n \in \mathbb{Z}}$ by $G$ are, respectively, denoted by

$$
\left\{\widetilde{h}_{n}\right\}_{n \in \mathbb{Z}}=\mathrm{G}\left\{h_{n}\right\}_{n \in \mathbb{Z}}, \quad\left\{\widetilde{h_{n}^{\prime}}\right\}_{n \in \mathbb{Z}}=\mathrm{G}^{-}\left\{h_{n}\right\}_{n \in \mathbb{Z}}
$$

By directly calculating, we have the following results.

Lemma 2.2. If $\left\{h_{n}\right\}_{n \in \mathbb{Z}}$ is a one-dimensional CQF, then $\left\{\tilde{h}_{n}\right\}_{n \in \mathbb{Z}}=\mathbf{G}\left\{h_{n}\right\}_{n \in \mathbb{Z}}$ and $\left\{\widetilde{h_{n}}\right\}_{n \in \mathbb{Z}}=$ $\mathrm{G}^{-}\left\{h_{n}\right\}_{n \in \mathbb{Z}}$ are one-dimensional CQFs.

Lemma 2.3. If $\left\{\tilde{h}_{n}\right\}_{n \in \mathbb{Z}}=\mathbf{G}\left\{h_{n}\right\}_{n \in \mathbb{Z}}$, then $\left\{h_{n}\right\}_{n \in \mathbb{Z}}=\left(\mathbf{G}^{T}\right)^{-}\left\{\tilde{h}_{n}\right\}_{n \in \mathbb{Z}}$.

Therefore, for a one-dimensional CQF, different one-dimensional CQFs can be derived when we choose different orthogonal matrices $G$. For a sequence $\left\{s_{n}\right\}_{n \in \mathbb{Z}}$, letting $\Gamma=\left\{n: s_{n} \neq 0\right\}$, we call $\Gamma$ the support of $\left\{s_{n}\right\}_{n \in \mathbb{Z}}$. If $\left\{s_{n}\right\}_{n \in \mathbb{Z}}$ is a one-dimensional CQF and the support of it is in $\{0,1\}$, we call $\left\{s_{n}\right\}_{n \in \mathbb{Z}}$ a simple one-dimensional CQF.

Theorem 2.4. If $\left\{h_{n}\right\}_{n \in \mathbb{Z}}$ is a one-dimensional $C Q F$, it can be constructed from a simple one-dimensional CQF by a series of SUT. 
Proof. It only needs to prove that by a series of ISUT of $\left\{h_{n}\right\}_{n \in \mathbb{Z}}$, we can get a simple one-dimensional CQF.

Without loss of generality, we assume that the support of $\left\{h_{n}\right\}_{n \in \mathbb{Z}}$ is in $\{0,1,2, \ldots, 2 N-$ $1\}$ and $h_{0} h_{2 N-1} \neq 0$. We will prove this theorem by induction.

We see that the theorem is true for $N=1$. Assume it is true for the case $N \leq L$ ( $L \geq$ $1, L \in \mathbb{Z})$. We now prove it is true for the case $N=L+1$. Suppose that $\left\{h_{n}\right\}_{n \in \mathbb{Z}}$ is a onedimensional CQF and $h_{0} h_{2 L+1} \neq 0$. We denote that

$$
G=\left(\begin{array}{cc}
\cos \theta & \sin \theta \\
-\sin \theta & \cos \theta
\end{array}\right), \quad\left\{\tilde{h}_{n}\right\}_{n \in \mathbb{Z}}=\mathrm{G}\left\{h_{n}\right\}_{n \in \mathbb{Z}}
$$

where $\tan \theta=-h_{1} / h_{0}$. Recall that $\left\{h_{n}\right\}_{n \in \mathbb{Z}}$ is a one-dimensional CQF, that is, $\sum_{n \in \mathbb{Z}} h_{n} h_{n+2 m}=$ $\delta_{0, m}$, which implies that $h_{0} h_{2 L}+h_{1} h_{2 L+1}=0$. Therefore,

$$
\begin{gathered}
\tilde{h}_{-1}=\sin \theta h_{0}+\cos \theta h_{1}=-\cos \theta h_{1}+\cos \theta h_{1}=0, \\
\tilde{h}_{2 L}=\cos \theta h_{2 L}-\sin \theta h_{2 L+1}=\cos \theta h_{2 L}+\cos \theta \frac{h_{1}}{h_{0}} h_{2 L+1}=0 .
\end{gathered}
$$

It follows that the support of $\left\{\tilde{h}_{n}\right\}_{n \in \mathbb{Z}}$ is in $\{0,1,2, \ldots, 2 L-1\}$. Suppose that $-\pi / 2<\theta<\pi / 2$, then $\tilde{h}_{0} \neq 0$. It follows that there exists $t \in \mathbb{Z}(t \leq L)$, such that $\widetilde{h}_{0} \widetilde{h}_{2 t-1} \neq 0$. Furthermore, if $n \in \mathbb{Z} \backslash\{0,1,2, \ldots, 2 t-1\}$, then $\widetilde{h}_{n}=0$. By the hypothesis, the theorem is proved.

Theorem 2.4 shows that any one-dimensional orthogonal low-pass wavelet filter can be constructed by a series of SUT. Suppose that an orthogonal scaling function $\phi(x)$ satisfies (2.1). Then the sequence $\left\{h_{n}\right\}_{n \in \mathbb{Z}}$ is a one-dimensional CQF. By Theorem 2.4, we know that $\left\{h_{n}\right\}_{n \in \mathbb{Z}}$ can be constructed by a simple one-dimensional CQF and a series of $2 \times 2$ orthogonal matrices. For a real number $\theta \in \mathbb{R}$, we denote $G_{\theta}$ as the $2 \times 2$ orthogonal matrix:

$$
\left(\begin{array}{cc}
\cos \theta & \sin \theta \\
-\sin \theta & \cos \theta
\end{array}\right), \quad \theta \in \mathbb{R}
$$

Let $\left\{\mathscr{\ell}_{n}\right\}_{n \in \mathbb{Z}}^{\gamma}$ be a simple one-dimensional CQF such that

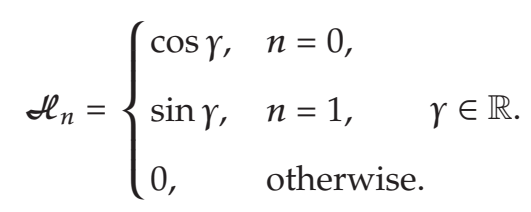

For an arbitrary positive integer $N$, choosing $\gamma_{0}, \gamma_{1}, \ldots, \gamma_{N-1} \in \mathbb{R}$, we define $\left\{h_{n}^{N}\right\}_{n \in \mathbb{Z}}$ as follows:

$$
\left\{h_{n}^{N}\right\}_{n \in \mathbb{Z}}=\mathbf{G}_{\gamma_{N-1}} \mathbf{G}_{\gamma_{N-2}} \cdots \mathbf{G}_{\gamma_{1}}\left\{\mathcal{L}_{n}\right\}_{n \in \mathbb{Z}}^{\gamma_{0}}
$$


In general, the support of $\left\{h_{n}^{N}\right\}_{n \in \mathbb{Z}}$ is in $[0,2 N-1] \cap \mathbb{Z}$. In other words, $\left\{h_{n}^{N}\right\}_{n \in \mathbb{Z}}$ is a length-2N filter. From the proof of Theorem 2.4, we know that the length- $2 N$ filter can be constructed by at most $N-1$ times SUT of one-dimensional CQF.

We can prove inductively the following theorems.

Theorem 2.5. Let $\eta=\gamma_{0}+\gamma_{1}+\cdots+\gamma_{N-1}$, then

$$
\sum_{i \in \mathbb{Z}} h_{2 i}^{N}=\cos \eta, \quad \sum_{i \in \mathbb{Z}} h_{2 i+1}^{N}=\sin \eta
$$

Theorem 2.6. Let

$$
\gamma_{0}+\gamma_{1}+\cdots+\gamma_{N-1}=2 k \pi+\frac{\pi}{4}, \quad k \in \mathbb{Z},
$$

then the sequence $\left\{h_{n}^{N}\right\}_{n \in \mathbb{Z}}$ given in (2.14) is a one-dimensional low-pass wavelet filter.

Theorem 2.6 provides a condition for choosing $\gamma_{0}, \gamma_{1}, \ldots, \gamma_{N-1}$ such that the one-dimensional CQF $\left\{h_{n}^{N}\right\}_{n \in \mathbb{Z}}$ given in (2.14) is a one-dimensional orthogonal low-pass wavelet filter.

In addition, if the low-pass wavelet filter $\left\{h_{n}^{N}\right\}_{n \in \mathbb{Z}}$ satisfies the Cohen's condition (see [4]), then the $\varphi(x)$ corresponding to $\left\{h_{n}^{N}\right\}_{n \in \mathbb{Z}}$ in (2.2) is an orthogonal scaling function.

By Theorem 2.6, we know that a length-2N one-dimensional low-pass wavelet filter $\left\{h_{n}^{N}\right\}_{n \in \mathbb{Z}}$ can be constructed by choosing $\gamma_{0}, \gamma_{1}, \ldots, \gamma_{N-1}$ such that condition (2.16) is satisfied. Therefore, any length- $2 N$ one-dimensional low-pass wavelet filter can be parameterized into a $(N-1)$-parameter family of wavelet system. In fact, we can give an explicit parametrization of any length-2N filter:

$$
\left\{h_{n}^{N}\right\}_{n \in \mathbb{Z}}=\mathbf{G}_{\gamma_{N-1}} \mathbf{G}_{\gamma_{N-2}} \cdots \mathbf{G}_{\gamma_{1}}\left\{\mathscr{\ell}_{n}\right\}_{n \in \mathbb{Z}}^{\pi / 4-\gamma_{N-1}-\gamma_{N-2} \cdots-\gamma_{1}}
$$

where $\gamma_{1}, \gamma_{2}, \ldots, \gamma_{N-1} \in \mathbb{R}$.

Applications of one-dimensional parameterized wavelets to compression are, for example, discussed in $[22,23]$. Parameterizing all possible filter coefficients that correspond to compactly supported one-dimensional orthonormal wavelets has been studied by several authors [6, 9, 24-26]. We provide explicit parametrization of any length- $2 N$ filters which satisfy the necessary conditions for orthogonality in terms of SUT.

\section{Construction of Two-Dimensional Wavelet Filters}

\subsection{SUT of Two-Dimensional CQF}

To construct two-dimensional orthogonal scaling function

$$
\phi(\mathbf{x})=2 \sum_{\alpha \in \mathbb{Z}^{2}} b_{\alpha} \phi(2 \mathbf{x}-\alpha)
$$


and its associated wavelets

$$
\psi^{l}(\mathbf{x})=2 \sum_{\alpha \in \mathbb{Z}^{2}} d_{\alpha}^{l} \phi(2 \mathbf{x}-\alpha), \quad l=1,2,3
$$

we need construct sequences $\left\{b_{\alpha}\right\}_{\alpha \in \mathbb{Z}^{2}}$ and $\left\{d_{\alpha}^{l}\right\}_{\alpha \in \mathbb{Z}^{2}}$ such that (see $[10,12,13]$, etc.)

$$
\begin{gathered}
\sum_{\alpha \in \mathbb{Z}^{2}} b_{\alpha}=2, \\
\sum_{\alpha \in \mathbb{Z}^{2}} b_{\alpha} b_{\alpha+2 \beta}=\delta_{0 \beta}, \\
\sum_{i, j \in \mathbb{Z}} b_{(2 i, 2 j)}=\sum_{i, j \in \mathbb{Z}} b_{(2 i+1,2 j)}=\sum_{i, j \in \mathbb{Z}} b_{(2 i, 2 j+1)}=\sum_{i, j \in \mathbb{Z}} b_{(2 i+1,2 j+1),} \\
\sum_{\alpha \in \mathbb{Z}^{2}} d_{\alpha}^{j_{1}} d_{\alpha+2 \beta}^{j_{2}}=\delta_{j_{1}, j_{2}} \delta_{0, \beta}, \\
\sum_{\alpha \in \mathbb{Z}^{2}} b_{\alpha} d_{\alpha+2 \beta}^{j}=0,
\end{gathered}
$$

where $j, j_{1}, j_{2}=1,2,3$ and $\beta \in \mathbb{Z}^{2}$.

The sequence $\left\{b_{\alpha}\right\}_{\alpha \in \mathbb{Z}^{2}}$, which satisfies (3.4), is called a two-dimensional CQF. If $\left\{b_{\alpha}\right\}_{\alpha \in \mathbb{Z}^{2}}$ satisfies (3.3), (3.4), and (3.5) simultaneously, we call it a two-dimensional low-pass wavelet filter. The sequences $\left\{d_{\alpha}^{j}\right\}_{\alpha \in \mathbb{Z}^{2}}(j=1,2,3)$, which satisfy (3.6) and (3.7), are called twodimensional high-pass wavelet filters.

For an arbitrary real-valued sequence $\left\{l_{\alpha}\right\}_{\alpha \in \mathbb{Z}^{2}} \in \ell^{2}\left(\mathbb{Z}^{2}\right)$, we define

$$
\Delta=\left\{\alpha: \alpha \in \mathbb{Z}^{2}, \quad l_{\alpha} \neq 0\right\}
$$

and $\Delta$ is called the support set of $\left\{l_{\alpha}\right\}_{\alpha \in \mathbb{Z}^{2}} \in \ell^{2}\left(\mathbb{Z}^{2}\right)$. If $\Delta$ is finite, we call $\left\{l_{\alpha}\right\}_{\alpha \in \mathbb{Z}^{2}} \in \ell^{2}\left(\mathbb{Z}^{2}\right)$ finite supported sequence. As aforementioned, the sequences we consider are real-valued and finite supported (FIR).

We note that any sequence $\left\{b_{\alpha}\right\}_{\alpha \in \mathbb{Z}^{2}}$ can be split into 4 disjoint subsets

$$
\begin{aligned}
\left\{b_{(2 i, 2 j)}: i, j \in \mathbb{Z}\right\}, & \left\{b_{(2 i, 2 j+1)}: i, j \in \mathbb{Z}\right\}, \\
\left\{b_{(2 i+1,2 j)}: i, j \in \mathbb{Z}\right\}, & \left\{b_{(2 i+1,2 j+1)}: i, j \in \mathbb{Z}\right\} .
\end{aligned}
$$


Definition 3.1. Let $U$ be an arbitrary $4 \times 4$ orthogonal matrix, and let $\left\{b_{\alpha}\right\}_{\alpha \in \mathbb{Z}^{2}}$ be an FIR. For integers $A_{s}, B_{s}(s=1,2,3,4)$ and for all $i, j \in \mathbb{Z}$, we set

$$
\begin{gathered}
\widetilde{\Gamma^{i, j}}=\left(\begin{array}{c}
\tilde{b}_{(2 i, 2 j)} \\
\tilde{b}_{(2 i, 2 j+1)} \\
\tilde{b}_{(2 i+1,2 j)} \\
\tilde{b}_{(2 i+1,2 j+1)}
\end{array}\right)^{T}, \\
\Gamma_{\left(A_{1}, B_{1}, A_{2}, B_{2}, A_{3}, B_{3}, A_{4}, B_{4}\right)}^{i, j}=\left(\begin{array}{c}
b_{\left(2 i-2 A_{1}, 2 j-2 B_{1}\right)} \\
b_{\left(2 i-2 A_{2}, 2 j-2 B_{2}+1\right)} \\
b_{\left(2 i-2 A_{3}+1,2 j-2 B_{3}\right)} \\
b_{\left(2 i-2 A_{4}+1,2 j-2 B_{4}+1\right)}
\end{array}\right)^{T} .
\end{gathered}
$$

Then $\left\{\tilde{b}_{\alpha}\right\}_{\alpha \in \mathbb{Z}^{2}}$, which is defined as follows:

$$
\widetilde{\Gamma^{i, j}}=\Gamma_{\left(A_{1}, B_{1}, A_{2}, B_{2}, A_{3}, B_{3}, A_{4}, B_{4}\right)}^{i, j} U
$$

is called the two-dimensional SUT of $\left\{b_{\alpha}\right\}_{\alpha \in \mathbb{Z}^{2}}$.

Lemma 3.2. If $\left\{b_{\alpha}\right\}_{\alpha \in \mathbb{Z}^{2}}$ is a two-dimensional CQF and $\left\{\tilde{b}_{\alpha}\right\}_{\alpha \in \mathbb{Z}^{2}}$ is given by (3.11), then $\left\{\tilde{b}_{\alpha}\right\}_{\alpha \in \mathbb{Z}^{2}}$ is also a two-dimensional CQF.

Proof. By directly calculating, we can prove that $\left\{\tilde{b}_{\alpha}\right\}_{\alpha \in \mathbb{Z}^{2}}$ satisfies the following equation:

$$
\sum_{\alpha \in \mathbb{Z}^{2}} \tilde{b}_{\alpha} \tilde{b}_{\alpha+2 \beta}=\delta_{0, \beta}, \quad \forall \beta \in \mathbb{Z}^{2}
$$

This completes the proof.

If the new two-dimensional CQF is a low-pass wavelet filter, then it is worthwhile to construct the associated high-pass wavelet filters. Now we provide a result of it.

Lemma 3.3. Suppose that $\left\{b_{\alpha}\right\}_{\alpha \in \mathbb{Z}^{2}}$ is a two-dimensional CQF, $\left\{d_{\alpha}^{k}\right\}_{\alpha \in \mathbb{Z}^{2}}(k=1,2,3)$ satisfy (3.6) and (3.7), $\left\{\widetilde{b}_{\alpha}\right\}_{\alpha \in \mathbb{Z}^{2}}$ is a two-dimensional CQF obtained by SUT of $\left\{b_{\alpha}\right\}_{\alpha \in \mathbb{Z}^{2}}$, then $\left\{\widetilde{d}_{\alpha}^{k}\right\}_{\alpha \in \mathbb{Z}^{2}}$, which are derived by SUT of $\left\{d_{\alpha}^{k}\right\}_{\alpha \in \mathbb{Z}^{2}}(k=1,2,3)$, satisfy (3.6) and (3.7) simultaneously.

It can be proved by direct calculation, so we omit the proof.

Definition 3.4. (i) $\{\tilde{b}\}_{\alpha \in \mathbb{Z}^{2}}$ is called the SUT0 of $\left\{b_{\alpha}\right\}_{\alpha \in \mathbb{Z}^{2}}$ if one chooses

$$
A_{i}=B_{i}=0 \quad(i=1,2,3,4)
$$

in (3.11). This transform is denoted by $\left\{\tilde{b}_{\alpha}\right\}_{\alpha \in \mathbb{Z}^{2}}={ }^{0} \mathbf{U}\left\{b_{\alpha}\right\}_{\alpha \in \mathbb{Z}^{2}}$. 
(ii) $\{\tilde{b}\}_{\alpha \in \mathbb{Z}^{2}}$ is called the SUTT1 of $\left\{b_{\alpha}\right\}_{\alpha \in \mathbb{Z}^{2}}$ if one chooses

$$
B_{2}=B_{4}=1, \quad B_{1}=B_{3}=A_{i}=0 \quad(i=1,2,3,4)
$$

in (3.11). This transform is denoted by $\left\{\tilde{b}_{\alpha}\right\}_{\alpha \in \mathbb{Z}^{2}}={ }^{T 1} \mathbf{U}\left\{b_{\alpha}\right\}_{\alpha \in \mathbb{Z}^{2}} .\{\tilde{b}\}_{\alpha \in \mathbb{Z}^{2}}$ is called the SUTT2 of $\left\{b_{\alpha}\right\}_{\alpha \in \mathbb{Z}^{2}}$ if one chooses

$$
A_{3}=A_{4}=1, \quad A_{1}=A_{2}=B_{i}=0 \quad(i=1,2,3,4) .
$$

This transform is denoted by $\left\{\tilde{b}_{\alpha}\right\}_{\alpha \in \mathbb{Z}^{2}}={ }^{T 2} \mathbf{U}\left\{b_{\alpha}\right\}_{\alpha \in \mathbb{Z}^{2}}$.

(iii) $\{\tilde{b}\}_{\alpha \in \mathbb{Z}^{2}}$ is called the SUT1 of $\left\{b_{\alpha}\right\}_{\alpha \in \mathbb{Z}^{2}}$ if one chooses

$$
A_{2}=A_{4}=1, \quad A_{1}=A_{3}=B_{i}=0 \quad(i=1,2,3,4)
$$

in (3.11). This transform is denoted by $\left\{\tilde{b}_{\alpha}\right\}_{\alpha \in \mathbb{Z}^{2}}={ }^{1} \mathbf{U}\left\{b_{\alpha}\right\}_{\alpha \in \mathbb{Z}^{2}} \cdot\{\tilde{b}\}_{\alpha \in \mathbb{Z}^{2}}$ is called the SUT2 of $\left\{b_{\alpha}\right\}_{\alpha \in \mathbb{Z}^{2}}$ if we choose

$$
B_{3}=B_{4}=1, \quad B_{1}=B_{2}=A_{i}=0 \quad(i=1,2,3,4) .
$$

This transform is denoted by $\left\{\tilde{b}_{\alpha}\right\}_{\alpha \in \mathbb{Z}^{2}}={ }^{2} \mathbf{U}\left\{b_{\alpha}\right\}_{\alpha \in \mathbb{Z}^{2}}$.

For a two-dimensional CQF, when we choose different orthogonal matrices, many new two-dimensional CQFs can be obtained. It is obvious that, after SUT0, the support of the new two-dimensional CQF does not change. But it is different for SUTT1, SUTT2, SUT1, and SUT2. For example, the support of the two-dimensional CQF

$$
b_{\alpha}= \begin{cases}\frac{1}{4} & \text { if } \alpha=(0,0) \text { or }(1,1) \\ \frac{\sqrt{3}}{4} & \text { if } \alpha=(1,0) \text { or }(0,1) \\ 0 & \text { otherwise }\end{cases}
$$

is $\{(0,0),(0,1),(1,0),(1,1)\}$, and the support of the filter $\left\{\tilde{b}_{\alpha}\right\}_{\alpha \in \mathbb{Z}^{2}}={ }^{1} \mathbf{U}\left\{b_{\alpha}\right\}_{\alpha \in \mathbb{Z}^{2}}$ is $\{(0,0),(0,1)$, $(1,0),(1,1),(2,0),(2,1),(3,0),(3,1)\} \subset\left([0,3] \times[0,1] \cap \mathbb{Z}^{2}\right)$, where $U=\operatorname{diag}(H, H)$ and

$$
H=\left[\begin{array}{cc}
\frac{1}{2} & \frac{\sqrt{3}}{2} \\
-\frac{\sqrt{3}}{2} & \frac{1}{2}
\end{array}\right] .
$$

In general, for integers $N, M$, if the support of $\left\{b_{\alpha}\right\}_{\alpha \in \mathbb{Z}^{2}}$ is in $[0,2 N-1] \times[0,2 M-1] \cap \mathbb{Z}^{2}$, after SUT1 (or SUT2) the support of the new filter is in $[0,2 N+1] \times[0,2 M-1] \cap \mathbb{Z}^{2}$ (or in $\left.[0,2 N-1] \times[0,2 M+1] \cap \mathbb{Z}^{2}\right)$. If the support of $\left\{b_{\alpha}\right\}_{\alpha \in \mathbb{Z}^{2}}$ is $\{(0,0),(1,0),(0,1),(1,1)\}$, we call $\left\{b_{\alpha}\right\}_{\alpha \in \mathbb{Z}^{2}}$ a simple two-dimensional filter. 
We will adopt the following notations in the rest of this paper. For arbitrary $\xi_{0}, \lambda_{0}, \xi$, $\lambda \in R$, let $\left\{\boldsymbol{B}_{\alpha}\right\}_{\alpha \in \mathbb{Z}^{2}}^{\lambda_{0}, \xi_{0}}$ be the FIR defined as follows:

$$
B_{\alpha}= \begin{cases}\cos \xi_{0} \cos \lambda_{0}, & \alpha=(0,0) \\ \cos \xi_{0} \sin \lambda_{0}, & \alpha=(0,1) \\ \sin \xi_{0} \cos \lambda_{0}, & \alpha=(1,0) \\ \sin \xi_{0} \sin \lambda_{0}, & \alpha=(1,1) \\ 0, & \text { otherwise }\end{cases}
$$

Furthermore, let $\left\{\Phi_{\alpha}^{k}\right\}_{\alpha \in \mathbb{Z}^{2}}^{\lambda_{0} \xi^{\circ}}(k=1,2,3)$ be the filters as follows

$$
\begin{aligned}
& \Phi_{\alpha}^{1}= \begin{cases}\cos \xi_{0} \sin \lambda_{0}, & \alpha=(0,0) \\
-\cos \xi_{0} \cos \lambda_{0}, & \alpha=(0,1) \\
\sin \xi_{0} \sin \lambda_{0}, & \alpha=(1,0) \\
-\sin \xi_{0} \cos \lambda_{0}, & \alpha=(1,1) \\
0, & \text { otherwise }\end{cases} \\
& \Phi_{\alpha}^{2}= \begin{cases}\sin \xi_{0} \cos \lambda_{0}, & \alpha=(0,0) \\
\sin \xi_{0} \sin \lambda_{0}, & \alpha=(0,1) \\
-\cos \xi_{0} \cos \lambda_{0}, & \alpha=(1,0) \\
-\cos \xi_{0} \sin \lambda_{0}, & \alpha=(1,1) \\
0, & \text { otherwise }\end{cases} \\
& \Phi_{\alpha}^{3}= \begin{cases}\sin \xi_{0} \sin \lambda_{0}, & \alpha=(0,0) \\
-\sin \xi_{0} \cos \lambda_{0}, & \alpha=(0,1) \\
-\cos \xi_{0} \sin \lambda_{0}, & \alpha=(1,0) \\
\cos \xi_{0} \cos \lambda_{0}, & \alpha=(1,1) \\
0, & \text { otherwise }\end{cases}
\end{aligned}
$$


and let $U_{\lambda}, U_{\xi}$ be orthogonal matrices such that

$$
\begin{aligned}
& U_{\lambda}=\left(\begin{array}{cccc}
\cos \lambda & \sin \lambda & 0 & 0 \\
-\sin \lambda & \cos \lambda & 0 & 0 \\
0 & 0 & \cos \lambda & \sin \lambda \\
0 & 0 & -\sin \lambda & \cos \lambda
\end{array}\right), \\
& U_{\xi}=\left(\begin{array}{cccc}
\cos \xi & 0 & \sin \xi & 0 \\
0 & \cos \xi & 0 & \sin \xi \\
-\sin \xi & 0 & \cos \xi & 0 \\
0 & -\sin \xi & 0 & \cos \xi
\end{array}\right),
\end{aligned}
$$

respectively. Then $\left\{\boldsymbol{B}_{\alpha}\right\}_{\alpha \in \mathbb{Z}^{2}}^{\lambda_{0}, \xi_{0}}$ is a simple two-dimensional CQF, and $\left\{\boldsymbol{\Phi}_{\alpha}^{k}\right\}_{\alpha \in \mathbb{Z}^{2}}^{\lambda_{0}, \xi_{0}}$ satisfy (3.6) and (3.7).

From the special two-dimensional CQF $\left\{\boldsymbol{B}_{\alpha}\right\}_{\alpha \in \mathbb{Z}^{2}}^{\lambda_{0}, \xi_{0}}$, by SUTT1, SUTT2, SUT1, SUT2, and the matrices $U_{\imath}, U_{\xi}$, we can construct some new two-dimensional CQFs.

\subsection{Tensor-Product Wavelet Filters}

In this subsection, we will show that all tensor-product wavelet filters can be constructed by SUTT1 and SUTT2.

A two-dimensional low-pass wavelet filters $\left\{b_{\alpha}\right\}_{\alpha \in \mathbb{Z}^{2}}$ is called tensor-product wavelet filter if it satisfies the following equations:

$$
\begin{gathered}
b_{(2 i, 2 j)}=h_{2 i} \tilde{h}_{2 j}, \\
b_{(2 i, 2 j+1)}=h_{2 i} \tilde{h}_{2 j+1}, \\
b_{(2 i+1,2 j)}=h_{2 i+1} \tilde{h}_{2 j}, \\
b_{(2 i+1,2 j+1)}=h_{2 i+1} \tilde{h}_{2 j+1},
\end{gathered}
$$

where $\left\{h_{i}\right\}_{i \in \mathbb{Z}},\left\{\tilde{h}_{i}\right\}_{i \in \mathbb{Z}}$ are one-dimensional orthogonal low-pass wavelet filters.

Theorem 3.5. If $\left\{b_{\alpha}\right\}_{\alpha \in \mathbb{Z}^{2}}$ is a tensor-product low-pass wavelet filter, then, it can be constructed by SUTT1 and SUTT2 from $\left\{\mathbb{B}_{\alpha}\right\}_{\alpha \in \mathbb{Z}^{2}}^{\lambda_{0}, \xi_{0}}$. 
Proof. For all $i, j \in \mathbb{Z}$, it follows from (3.24) that

$$
\left(b_{(2 i, 2 j)}, b_{(2 i, 2 j+1)}, b_{(2 i+1,2 j)}, b_{(2 i+1,2 j+1)}\right)=\left(h_{2 i}, h_{2 i}, h_{2 i+1}, h_{2 i+1}\right)\left(\begin{array}{cccc}
\tilde{h}_{2 j} & 0 & 0 & 0 \\
0 & \tilde{h}_{2 j+1} & 0 & 0 \\
0 & 0 & \tilde{h}_{2 j} & 0 \\
0 & 0 & 0 & \tilde{h}_{2 j+1}
\end{array}\right) .
$$

Suppose that $\left\{\tilde{h}_{i}\right\}_{i \in \mathbb{Z}}$ is a length- $2 N$ one-dimensional filter. Then there exist $\lambda_{0}, \ldots, \lambda_{N-2}$, $\lambda_{N-1} \in R$ such that $\lambda_{0}+\cdots+\lambda_{N-2}+\lambda_{N-1}=\pi / 4$, and

$$
\left\{\tilde{h}_{n}\right\}_{n \in \mathbb{Z}}=\mathrm{G}_{\lambda_{N-1}} \mathrm{G}_{\lambda_{N-2}} \cdots \mathrm{G}_{\lambda_{1}}\left\{\mathscr{L}_{n}\right\}_{n \in \mathbb{Z}}^{\lambda_{0}}
$$

We denote

$$
\left\{\tilde{h}_{n}\right\}_{n \in \mathbb{Z}}^{N-1}=\mathrm{G}_{\lambda_{N-2}} \cdots \mathrm{G}_{\lambda_{1}}\left\{\mathscr{\ell}_{n}\right\}_{n \in \mathbb{Z}}^{\lambda_{0}}
$$

Let $\left\{b_{\alpha}\right\}_{\alpha \in \mathbb{Z}^{2}}^{N-1}$ be two-dimensional filter such that

$$
\begin{gathered}
b_{(2 i, 2 j)}^{N-1}=h_{2 i} \tilde{h}_{2 j}^{N-1}, \\
b_{(2 i, 2 j+1)}^{N-1}=h_{2 i} \widetilde{h}_{2 j+1}^{N-1}, \\
b_{(2 i+1,2 j)}^{N-1}=h_{2 i+1} \widetilde{h}_{2 j}^{N-1}, \\
b_{(2 i+1,2 j+1)}^{N-1}=h_{2 i+1} \widetilde{h}_{2 j+1}^{N-1},
\end{gathered}
$$

It follows that

$$
\begin{aligned}
& \left(b_{(2 i, 2 j)}, b_{(2 i, 2 j+1)}, b_{(2 i+1,2 j)}, b_{(2 i+1,2 j+1)}\right) \\
& \quad=\left(h_{2 i}, h_{2 i}, h_{2 i+1}, h_{2 i+1}\right)\left(\begin{array}{cccc}
\tilde{h}_{2 j} & 0 & 0 & 0 \\
0 & \tilde{h}_{2 j+1} & 0 & 0 \\
0 & 0 & \tilde{h}_{2 j} & 0 \\
0 & 0 & 0 & \tilde{h}_{2 j+1}
\end{array}\right)
\end{aligned}
$$




$$
\begin{aligned}
& =\left(h_{2 i}, h_{2 i}, h_{2 i+1}, h_{2 i+1}\right)\left(\begin{array}{cccc}
\tilde{h}_{2 j}^{N-1} & 0 & 0 & 0 \\
0 & \tilde{h}_{2 j-1}^{N-1} & 0 & 0 \\
0 & 0 & \tilde{h}_{2 j}^{N-1} & 0 \\
0 & 0 & 0 & \tilde{h}_{2 j-1}^{N-1}
\end{array}\right) \\
& \times\left(\begin{array}{cccc}
\cos \lambda_{N-1} & \sin \lambda_{N-1} & 0 & 0 \\
-\sin \lambda_{N-1} & \cos \lambda_{N-1} & 0 & 0 \\
0 & 0 & \cos \lambda_{N-1} & \sin \lambda_{N-1} \\
0 & 0 & -\sin \lambda_{N-1} & \cos \lambda_{N-1}
\end{array}\right) \\
& =\left(b_{(2 i, 2 j)}^{N-1}, b_{(2 i, 2 j-1)}^{N-1}, b_{(2 i+1,2 j)}^{N-1}, b_{(2 i+1,2 j-1)}^{N-1}\right) U_{\lambda_{N-1} .}
\end{aligned}
$$

Hence, $\left\{b_{\alpha}\right\}_{\alpha \in \mathbb{Z}^{2}}$ can be constructed by SUTT1 of $\left\{b_{\alpha}\right\}_{\alpha \in \mathbb{Z}^{2}}^{N-1}$.

It can be proved inductively that $\left\{b_{\alpha}\right\}_{\alpha \in \mathbb{Z}^{2}}$ can be constructed by a series of SUTT1 from $\left\{b_{\alpha}\right\}_{\alpha \in \mathbb{Z}^{2}}^{0}$, and $\left\{b_{\alpha}\right\}_{\alpha \in \mathbb{Z}^{2}}^{0}$ can be constructed by a series of SUTT2 from $\left\{B_{\alpha}\right\}_{\alpha \in \mathbb{Z}^{2}}^{\alpha_{0}, \xi_{0}^{0}}$, where $\left\{b_{\alpha}\right\}_{\alpha \in \mathbb{Z}^{2}}^{0}$ denotes the filter

$$
\begin{aligned}
b_{(2 i, 2 j)}^{0} & =h_{2 i} \widetilde{\mathscr{\ell}}_{2 j}, \\
b_{(2 i, 2 j+1)}^{0} & =h_{2 i} \widetilde{\mathscr{\ell}}_{2 j+1}, \\
b_{(2 i+1,2 j)}^{0} & =h_{2 i+1} \widetilde{\mathscr{l}}_{2 j}, \quad i, j \in \mathbb{Z} . \\
b_{(2 i+1,2 j+1)}^{0} & =h_{2 i+1} \widetilde{\mathscr{\ell}}_{2 j+1},
\end{aligned}
$$

Therefore, any tensor-product two-dimensional low-pass wavelet filters can be constructed by SUTT1 and SUTT2 from $\left\{\boldsymbol{B}_{\alpha}\right\}_{\alpha \in \mathbb{Z}^{2}}^{\Lambda_{0}, \xi_{0}}$.

\subsection{Two-Dimensional Wavelet Filters in Terms of SUT1 and SUT2}

From now on, we give a method of construction of two-dimensional orthogonal wavelet filters from a simple two-dimensional CQF by SUT1 and SUT2.

For arbitrary positive integers $N$ and $M$, choosing $\lambda_{0}, \lambda_{1}, \ldots, \lambda_{N-1}, \xi_{0}, \xi_{1}, \ldots, \xi_{M-1} \in \mathbb{R}$, $\left\{b_{\alpha}^{N, M}\right\}_{\alpha \in \mathbb{Z}^{2}}$ is defined as

$$
\left\{b_{\alpha}^{N, M}\right\}_{\alpha \in \mathbb{Z}^{2}}=\varepsilon_{n_{N+M-2}} U_{n_{N+M-2}} \varepsilon_{n_{N+M-3}} U_{n_{N+M-3}} \ldots \varepsilon_{n_{1}} U_{n_{1}}\left\{B_{\alpha}\right\}_{\alpha \in \mathbb{Z}^{2}}^{\lambda_{0}, \xi_{0}}
$$


where $n_{1}, n_{2}, \ldots, n_{N+M-2} \in\left\{\lambda_{1}, \lambda_{2}, \ldots, \lambda_{N-1}, \xi_{1}, \xi_{2}, \ldots, \xi_{M-1}\right\}$ and

$$
\varepsilon_{n_{j}}= \begin{cases}1, & \text { if } n_{j} \in\left\{\lambda_{1}, \lambda_{2}, \ldots, \lambda_{N-1}\right\} \\ 2, & \text { if } n_{j} \in\left\{\xi_{1}, \xi_{2}, \ldots, \xi_{M-1}\right\} .\end{cases}
$$

Then $\left\{b_{\alpha}^{N, M}\right\}_{\alpha \in \mathbb{Z}^{2}}$ is a two-dimensional CQF, and its support is in $[0,2 N-1] \times[0,2 M-1] \cap \mathbb{Z}^{2}$. We are now in a position to draw some conditions on choosing $\lambda_{0}, \lambda_{1}, \ldots, \lambda_{N_{1}}, \xi_{0}, \xi_{1}, \ldots, \xi_{M_{1}}$, such that $\left\{b_{\alpha}^{N, M}\right\}_{\alpha \in \mathbb{Z}^{2}}$ is a two-dimensional low-pass wavelet filter.

Theorem 3.6. Let $\eta=\lambda_{0}+\lambda_{1}+\cdots+\lambda_{N-1}, \quad \tilde{\eta}=\xi_{0}+\xi_{1}+\cdots+\xi_{M-1}$. Then

$$
\begin{aligned}
\sum_{i, j \in \mathbb{Z}} b_{(2 i, 2 j)}^{N, M}=\cos \eta \cos \tilde{\eta}, & \sum_{i, j \in \mathbb{Z}} b_{(2 i, 2 j+1)}^{N, M}=\sin \eta \cos \tilde{\eta}, \\
\sum_{i, j \in \mathbb{Z}} b_{(2 i+1,2 j)}^{N, M}=\cos \eta \sin \tilde{\eta}, & \sum_{i, j \in \mathbb{Z}} b_{(2 i+1,2 j+1)}^{N, M}=\sin \eta \sin \tilde{\eta} .
\end{aligned}
$$

Proof. It can be proved inductively. For the case $N=M=1$, it is obviously true. Assume that it is true for the case $N \leq k_{1}, M \leq k_{2}\left(k_{1} \geq 1, k_{2} \geq 1, k_{1}, k_{2} \in \mathbb{Z}\right)$; now we prove that it is true for the case $N=k_{1}+1, M=k_{2}$.

Let $s$ be the integer such that $n_{s+1} \in\left\{\lambda_{1}, \lambda_{2}, \ldots, \lambda_{k_{1}}\right\}$ and $n_{s+t} \in\left\{\xi_{1}, \xi_{2}, \ldots, \xi_{k_{2}}\right\}(t>1, t \in$ $\mathbb{Z})$. Suppose that

$$
\begin{gathered}
i_{1}, i_{2}, \ldots, i_{u} \leq s \Longrightarrow n_{i_{1}}, n_{i_{2}}, \ldots, n_{i_{u}} \in\left\{\lambda_{1}, \lambda_{2}, \ldots, \lambda_{k_{1}}\right\} \\
j_{1}, j_{2}, \ldots, j_{v} \leq s \Longrightarrow n_{j_{1}}, n_{j_{2}}, \ldots, n_{j_{v}} \in\left\{\xi_{1}, \xi_{2}, \ldots, \xi_{k_{1}}\right\} .
\end{gathered}
$$

It follows that $u=k_{1}$ and $v \leq k_{2}-1$. Let

$$
\begin{gathered}
\eta_{s}=\lambda_{0}+n_{i_{1}}+n_{i_{2}}+\cdots+n_{i_{u}}, \quad \tilde{\eta}_{s}=\xi_{0}+n_{j_{1}}+n_{j_{2}}+\cdots+n_{j_{v}}, \\
\left\{b_{\alpha}^{s}\right\}_{\alpha \in \mathbb{Z}^{2}}={ }^{\varepsilon_{n_{s}}} U_{n_{s}}{ }^{\varepsilon_{n_{s-1}}} U_{n_{s-1}} \ldots{ }^{\varepsilon_{n_{1}}} U_{n_{1}}\left\{B_{\alpha}\right\}_{\alpha \in \mathbb{Z}^{2}}^{\lambda_{0}, \xi^{\circ}} ;
\end{gathered}
$$

it follows that

$$
\begin{aligned}
\sum_{i, j \in \mathbb{Z}} b_{(2 i, 2 j)}^{s} & =\cos \eta_{s} \cos \tilde{\eta}_{s}, & \sum_{i, j \in \mathbb{Z}} b_{(2 i, 2 j+1)}^{s}=\sin \eta_{s} \cos \tilde{\eta}_{s} \\
\sum_{i, j \in \mathbb{Z}} b_{(2 i+1,2 j)}^{s} & =\cos \eta_{s} \sin \tilde{\eta}_{s}, & \sum_{i, j \in \mathbb{Z}} b_{(2 i+1,2 j+1)}^{s}=\sin \eta_{s} \sin \tilde{\eta}_{s} .
\end{aligned}
$$


Let $\left\{b_{\alpha}^{s+1}\right\}_{\alpha \in \mathbb{Z}^{2}}=\varepsilon_{n_{s+1}} U_{n_{s+1}}\left\{b_{\alpha}^{s}\right\}_{\alpha \in \mathbb{Z}^{2}}$. Since $n_{s+1} \in\left\{\lambda_{1}, \lambda_{2}, \ldots, \lambda_{k_{1}}\right\}$, then

$$
\begin{gathered}
b_{(2 i, 2 j)}^{s+1}=\cos n_{s+1} b_{(2 i, 2 j)}^{s}-\sin n_{s+1} b_{(2 i-2,2 j+1)^{\prime}}^{s} \\
b_{(2 i, 2 j+1)}^{s+1}=\sin n_{s+1} b_{(2 i, 2 j)}^{s}+\cos n_{s+1} b_{(2 i-2,2 j+1)^{\prime}}^{s} \\
b_{(2 i+1,2 j)}^{s+1}=\cos n_{s+1} b_{(2 i+1,2 j)}^{s}-\sin n_{s+1} b_{(2 i-1,2 j+1)^{\prime}}^{s} \\
b_{(2 i+1,2 j+1)}^{s+1}=\sin n_{s+1} b_{(2 i+1,2 j)}^{s}+\cos n_{s+1} b_{(2 i-1,2 j+1)^{s}}^{s}
\end{gathered}
$$

Therefore,

$$
\begin{array}{cc}
\sum_{i, j \in \mathbb{Z}} b_{(2 i, 2 j)}^{s+1}=\cos \left(\eta_{s}+n_{s+1}\right) \cos \tilde{\eta}_{s} ; & \sum_{i, j \in \mathbb{Z}} b_{(2 i, 2 j+1)}^{s+1}=\sin \left(\eta_{s}+n_{s+1}\right) \cos \tilde{\eta}_{s} ; \\
\sum_{i, j \in \mathbb{Z}} b_{(2 i+1,2 j)}^{s+1}=\cos \left(\eta_{s}+n_{s+1}\right) \sin \tilde{\eta}_{s} ; & \sum_{i, j \in \mathbb{Z}} b_{(2 i+1,2 j+1)}^{s+1}=\sin \left(\eta_{s}+n_{s+1}\right) \sin \tilde{\eta}_{s} .
\end{array}
$$

For $t>1$, let

$$
\left\{b_{\alpha}^{s+t}\right\}_{\alpha \in \mathbb{Z}^{2}}={ }^{\varepsilon_{n_{s+t}}} U_{n_{s+t}} \ldots \varepsilon_{n_{s+1}} U_{n_{s+1}}\left\{b_{\alpha}^{s}\right\}_{\alpha \in \mathbb{Z}^{2}} .
$$

It follows from $n_{s+t} \in\left\{\xi_{1}, \xi_{2}, \ldots, \xi_{k_{2}-1}\right\}$ that

$$
\begin{gathered}
b_{(2 i, 2 j)}^{s+t}=\cos n_{s+t} b_{(2 i, 2 j)}^{s+t-1}-\sin n_{s+t} b_{(2 i+1,2 j-2)^{\prime}}^{s+t-1} \\
b_{(2 i, 2 j+1)}^{s+t}=\cos n_{s+t} b_{(2 i, 2 j+1)}^{s+t-1}-\sin n_{s+t} b_{(2 i+1,2 j-1)^{\prime}}^{s+t-1} \\
b_{(2 i+1,2 j)}^{s+t}=\sin n_{s+t} b_{(2 i, 2 j)}^{s+t-1}+\cos n_{s+t} b_{(2 i+1,2 j-2)^{\prime}}^{s+t-1} \\
b_{(2 i+1,2 j+1)}^{s+t}=\sin n_{s+t} b_{(2 i, 2 j+1)}^{s+t-1}+\cos n_{s+t} b_{(2 i+1,2 j-1)}^{s+t-1} .
\end{gathered}
$$

Therefore,

$$
\begin{aligned}
& \sum_{i, j \in \mathbb{Z}} b_{(2 i, 2 j)}^{s+t}=\cos \eta_{s+t-1} \cos \left(\tilde{\eta}_{s+t-1}+n_{s+t}\right) \\
& \sum_{i, j \in \mathbb{Z}} b_{(2 i, 2 j+1)}^{s+t}=\sin \eta_{s+t-1} \cos \left(\tilde{\eta}_{s+t-1}+n_{s+t}\right) \\
& \sum_{i, j \in \mathbb{Z}} b_{(2 i+1,2 j)}^{s+t}=\cos \eta_{s+t-1} \sin \left(\tilde{\eta}_{s+t-1}+n_{s+t}\right) \\
& \sum_{i, j \in \mathbb{Z}} b_{(2 i+1,2 j+1)}^{s+t}=\sin \eta_{s+t-1} \sin \left(\tilde{\eta}_{s+t-1}+n_{s+t}\right),
\end{aligned}
$$


where

$$
\eta_{s+t-1}=\lambda_{0}+\lambda_{1}+\cdots+\lambda_{k_{1}}, \quad \tilde{\eta}_{s+t-1}=\xi_{0}+n_{j_{1}}+\cdots+n_{j_{v}}+n_{s+2}+n_{s+t-1} .
$$

Namely, it is true for the case $N=k_{1}+1, M=k_{2}$.

Similarly for the case $N=k_{1}, M=k_{2}+1$. This completes the proof.

Now we provide the condition on choosing $\lambda_{0}, \lambda_{1}, \ldots, \lambda_{N_{1}}, \xi_{0}, \xi_{1}, \ldots, \xi_{M_{1}}$, such that $\left\{b_{\alpha}^{N, M}\right\}_{\alpha \in \mathbb{Z}^{2}}$ is a two-dimensional low-pass wavelet filter.

Theorem 3.7. If there exists integers $n_{1}$ and $n_{2}$, such that

$$
\begin{aligned}
& \eta=\lambda_{0}+\lambda_{1}+\cdots+\lambda_{N-1}=2 n_{1} \pi+\frac{\pi}{4}, \\
& \tilde{\eta}=\xi_{0}+\xi_{1}+\cdots+\xi_{M-1}=2 n_{2} \pi+\frac{\pi}{4},
\end{aligned}
$$

then the sequence $\left\{b_{\alpha}^{N, M}\right\}_{\alpha \in \mathbb{Z}^{2}}$ constructed in (3.31) is a two-dimensional low-pass wavelet filter.

Proof. It follows from Theorem 3.6 that

$$
\begin{array}{ll}
\sum_{i, j \in \mathbb{Z}} b_{(2 i, 2 j)}=\cos \eta \cos \tilde{\eta}=\frac{1}{2}, \quad & \sum_{i, j \in \mathbb{Z}} b_{(2 i, 2 j+1)}=\sin \eta \cos \tilde{\eta}=\frac{1}{2} \\
\sum_{i, j \in \mathbb{Z}} b_{(2 i+1,2 j)}=\cos \eta \sin \tilde{\eta}=\frac{1}{2}, \quad \sum_{i, j \in \mathbb{Z}} b_{(2 i+1,2 j+1)}=\sin \eta \sin \tilde{\eta}=\frac{1}{2} .
\end{array}
$$

Therefore, $\left\{b_{\alpha}^{N, M}\right\}_{\alpha \in \mathbb{Z}^{2}}$ satisfies conditions (3.3) and (3.5), then it is a two-dimensional lowpass wavelet filter.

Corollary 3.8. If $\left\{b_{\alpha}^{N, M}\right\}_{\alpha \in \mathbb{Z}^{2}}$ as given in (3.31) is a low-pass wavelet filter, then

$$
\left\{d_{\alpha}^{k, N, M}\right\}_{\alpha \in \mathbb{Z}^{2}}={ }^{\varepsilon_{n_{N+M-2}}} U_{n_{N+M-2}} \varepsilon_{n_{N+M-3}} U_{n_{N+M-3}} \ldots \varepsilon_{n_{1}} U_{n_{1}}\left\{\Phi_{\alpha}^{k}\right\}_{\alpha \in \mathbb{Z}^{2}}^{\Lambda_{0}, \xi_{0}}
$$

are the high-pass filters associated with $\left\{b_{\alpha}^{N, M}\right\}_{\alpha \in \mathbb{Z}^{2}}$, where $k=1,2,3$. 
Table 1: The coefficients of a nonseparable orthogonal low-pass wavelet filter.

\begin{tabular}{lcccc}
\hline$b_{(i, j)}$ & $j=0$ & $j=1$ & $j=2$ & $j=3$ \\
\hline$i=0$ & -0.020275 & -0.054787 & 0.243250 & 0.657306 \\
$i=1$ & 0.024899 & 0.067282 & 0.198076 & 0.535237 \\
$i=2$ & -0.025190 & 0.009322 & 0.302214 & -0.111841 \\
$i=3$ & 0.030935 & -0.011448 & 0.246090 & -0.091071 \\
\hline
\end{tabular}

Table 2: The coefficients of a high-pass wavelet filter $\left(d_{(i, j)}^{1}\right)$ associated with the low-pass wavelet filter in Table 1.

\begin{tabular}{lcccc}
\hline$d_{(i, j)}^{1}$ & $j=0$ & $j=1$ & $j=2$ & $j=3$ \\
\hline$i=0$ & 0.009322 & 0.025190 & -0.111841 & -0.302214 \\
$i=1$ & -0.011448 & -0.030935 & -0.091071 & -0.246090 \\
$i=2$ & -0.054787 & 0.020275 & 0.657306 & -0.243250 \\
$i=3$ & 0.067282 & -0.024899 & 0.535237 & -0.198076 \\
\hline
\end{tabular}

Remark 3.9. We can choose other orthogonal matrices than $U_{\lambda}$ and $U_{\xi}$ such as

$$
\begin{aligned}
& U_{\lambda, \tilde{\lambda}}=\left(\begin{array}{cccc}
\cos \lambda & \sin \lambda & 0 & 0 \\
-\sin \lambda & \cos \lambda & 0 & 0 \\
0 & 0 & \cos \tilde{\lambda} & \sin \tilde{\lambda} \\
0 & 0 & -\sin \tilde{\lambda} & \cos \tilde{\lambda}
\end{array}\right), \\
& U_{\xi, \tilde{\xi}}=\left(\begin{array}{cccc}
\cos \xi & 0 & \sin \xi & 0 \\
0 & \cos \tilde{\xi} & 0 & \sin \tilde{\xi} \\
-\sin \xi & 0 & \cos \xi & 0 \\
0 & -\sin \tilde{\xi} & 0 & \cos \tilde{\xi}
\end{array}\right),
\end{aligned}
$$

where $\lambda, \tilde{\lambda}, \xi, \tilde{\xi} \in \mathbb{R}$; but conditions in Theorem 3.7 should be changed.

Example 3.10. We choose $N=2, M=2$ in (3.31). For $\xi_{0}, \xi_{1}, \lambda_{0}, \lambda_{1} \in \mathbb{R}$, define $\left\{b_{\alpha}\right\}_{\alpha \in \mathbb{Z}^{2}}$ as follows:

$$
\left\{b_{\alpha}\right\}_{\alpha \in \mathbb{Z}^{2}}={ }^{2} U_{\xi_{1}}{ }^{1} U_{\lambda_{1}}\left\{B_{\alpha}\right\}_{\alpha \in \mathbb{Z}^{2}}^{\lambda_{0, \xi_{0}}} .
$$

By choosing $\xi_{0}, \xi_{1}, \lambda_{0}, \lambda_{1}$ such that $\lambda_{0}+\lambda_{1}=\xi_{0}+\xi_{1}=\pi / 4$, we can get many twodimensional low-pass wavelet filters and their corresponding high-pass wavelet filters. For instance, set $\xi_{1}=2.254190, \xi_{0}=\pi / 4-\xi_{1}, \lambda_{1}=4.357946, \lambda_{0}=\pi / 4-\lambda_{1}$. By (3.31) and (3.47), we can get a nonseparable orthogonal low-pass wavelet filter (see Table 1) and its associated high-pass wavelet filters (Tables 2, 3, and 4). Figure 1 shows that the high frequency subbands by the derived filter can reveal more features than that by the commonly used tensorproduct wavelet filter.

Remark 3.11. By Section 3.2, we know that any two-dimensional tensor-product orthogonal wavelet filters can be constructed by SUTT1 and SUTT2. By Example 3.10, we know that, by 


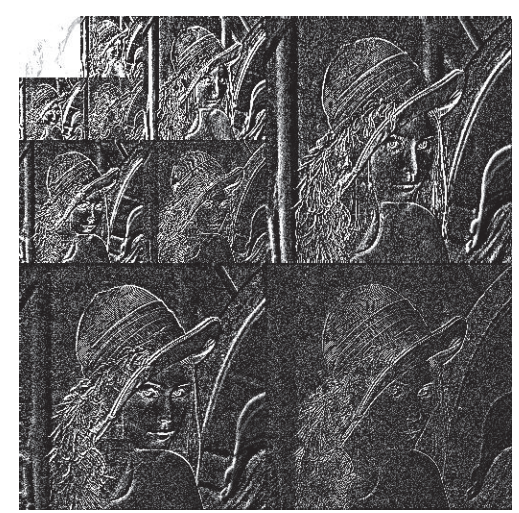

(a)

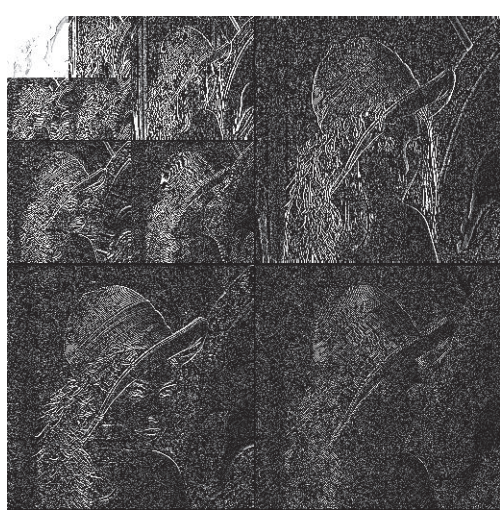

(b)

Figure 1: It shows that the high-frequency subbands by the derived filter can reveal more features than that by the commonly used tensor-product wavelet filter. (a) Decomposition of the "Lena" image by the derived filter. (b) Decomposition of the "Lena" image by tensor-product filter. All the coefficients in the high-frequency subbands are magnified by a factor 20 to see the difference.

Table 3: The coefficients of a high-pass wavelet filter $\left(d_{(i, j)}^{2}\right)$ associated with the low-pass wavelet filter in Table 1.

\begin{tabular}{ccccc}
\hline$d_{(i, j)}^{2}$ & $j=0$ & $j=1$ & $j=2$ & $j=3$ \\
\hline$i=0$ & 0.198076 & 0.535237 & 0.024899 & 0.067282 \\
$i=1$ & -0.243250 & -0.657306 & 0.020275 & 0.054787 \\
$i=2$ & 0.246090 & -0.091071 & 0.030935 & -0.011448 \\
$i=3$ & -0.302214 & 0.111841 & 0.025190 & -0.009322 \\
\hline
\end{tabular}

Table 4: The coefficients of a high-pass wavelet filter $\left(d_{(i, j)}^{3}\right)$ associated with the low-pass wavelet filter in Table 1.

\begin{tabular}{lcccc}
\hline$d_{(i, j)}^{3}$ & $j=0$ & $j=1$ & $j=2$ & $j=3$ \\
\hline$i=0$ & -0.091071 & -0.246090 & -0.011448 & -0.030935 \\
$i=1$ & 0.111841 & 0.302214 & -0.009322 & -0.025190 \\
$i=2$ & 0.535237 & -0.198076 & 0.067282 & -0.024899 \\
$i=3$ & -0.657306 & 0.243250 & 0.054787 & -0.020275 \\
\hline
\end{tabular}

SUT1 and SUT2, nonseparable wavelet filters can be achieved. Therefore, the construction of two-dimensional wavelet filters in terms of SUT of two-dimensional CQF is a generalization of the construction of separable orthogonal wavelet filters. Furthermore, from (3.31) and (3.47), we can see that our construction is a parametrization method.

\section{Conclusion}

SUT of CQF is introduced in this paper. In terms of SUT of one-dimensional CQF, any one-dimensional orthogonal wavelet filters with dilation factor 2 can be given in explicit expression. The SUT of two-dimensional CQF is applied to the construction of two-dimensional orthogonal wavelet filters, and a parametrization method is presented. The selection 
of the parameters is not restricted by any implicit condition. Tensor-product wavelet filters are only special case of this method. It provides more ways to randomly generate perfect reconstruction filters.

Our method provides many possible choices for the parameters. But what is a good choice of the parameters? Should any restriction on the choice of the parameters imply certain properties? Characteristics of SUT should be deeply studied.

\section{Acknowledgments}

The authors would like to thank the anonymous reviewers and the associate editor for their valuable comments and suggestions to improve the presentation of this paper. This work was supported in part by the National Science Foundation of China under Grant 60973157. This work was also supported in part by the National Science Foundation of Jiangsu Province Education Department under Grant no. 08KJB520004, and in part by Philosophy and Social Sciences Planning Project of Beijing Municipal Commission of Education under Grant no. SM201010011002.

\section{References}

[1] I. Daubechies, "Orthonormal bases of compactly supported wavelets," Communications on Pure and Applied Mathematics, vol. 41, no. 7, pp. 909-996, 1988.

[2] M. Vetterli, "Wavelets, approximation, and compression," IEEE Signal Processing Magazine, vol. 18, no. 5, pp. 59-73, 2001.

[3] A. Cohen, I. Daubechies, and J. C. Feauveau, "Biorthogonal bases of compactly supported wavelets," Communications on Pure and Applied Mathematics, vol. 45, no. 5, pp. 485-560, 1992.

[4] I. Daubechies, Ten Lectures on Wavelets, vol. 61 of CBMS-NSF Regional Conference Series in Applied Mathematics, Society for Industrial and Applied Mathematics (SIAM), Philadelphia, Pa, USA, 1992.

[5] P. N. Heller, "Rank $m$ wavelets with $n$ vanishing moments," SIAM Journal on Matrix Analysis and Applications, vol. 16, no. 2, pp. 502-519, 1995.

[6] G. Regensburger, "Parametrizing compactly supported orthonormal wavelets by discrete moments," Applicable Algebra in Engineering, Communication and Computing, vol. 18, no. 6, pp. 583-601, 2007.

[7] H. L. Resnikoff, J. Tian, and R. O. Wells, Jr., "Biorthogonal wavelet space: parametrization and factorization," SIAM Journal on Mathematical Analysis, vol. 33, no. 1, pp. 194-215, 2001.

[8] W. Sweldens, "The lifting scheme: a custom-design construction of biorthogonal wavelets," Applied and Computational Harmonic Analysis, vol. 3, no. 2, pp. 186-200, 1996.

[9] P. P. Vaidyananathan, Multirate Systems and Filter Banks, Prentice-Hall, Cliffs, NJ, USA, 1993.

[10] A. Ayache, "Some methods for constructing nonseparable, orthonormal, compactly supported wavelet bases," Applied and Computational Harmonic Analysis, vol. 10, no. 1, pp. 99-111, 2001.

[11] E. Belogay and Y. Wang, "Arbitrarily smooth orthogonal nonseparable wavelets in $R^{2}$," SIAM Journal on Mathematical Analysis, vol. 30, no. 3, pp. 678-697, 1999.

[12] W. He and M. J. Lai, "Examples of bivariate nonseparable compactly supported orthonormal continuous wavelets," IEEE Transactions on Image Processing, vol. 9, no. 5, pp. 949-953, 2000.

[13] M. J. Lai, "Construction of multivariate compactly supported orthonormal wavelets," Advances in Computational Mathematics, vol. 25, no. 1-3, pp. 41-56, 2006.

[14] A. Cohen and I. Daubechies, "Nonseparable bidimensional wavelet bases," Revista Matemática Iberoamericana, vol. 9, no. 1, pp. 51-137, 1993.

[15] J. Kovačević and M. Vetterli, "Nonseparable multidimensional perfect reconstruction filter banks," IEEE Transactions on Information Theory, vol. 38, no. 2, part 2, pp. 533-555, 1992.

[16] G. Strang and T. Nguyen, Wavelets and Filter Banks, Wellesley-Cambridge Press, Wellesley, Mass, USA, 1996.

[17] Y. W. Wang, J. F. Doherty, and R. E. Van Dyck, “A waveletbased watermarking algorithm for ownership verification of digital image," IEEE Transactions on Image Processing, vol. 11, no. 2, pp. 77$88,2002$. 
[18] W. Dietl, P. Meerwald, and A. Uhl, "Protection of waveletbased watermarking systems using filter parametrization," Signal Processing, vol. 83, no. 10, pp. 2095-2116, 2003.

[19] Z. Huang and Z. Jiang, "Image ownership verification via private pattern and watermarking wavelet filters," in Proceedings of the 7th Digital Image Computing: Techniques and Applications, C. Sun, H. Talbot, S. Ourselin, and T. Adriaansen, Eds., pp. 801-810, Sydney, Australia, December 2003.

[20] P. Meerwald and A. Uhl, "A survey of wavelet-domain watermarking algorithms," in Electronic Imaging, Security and Watermarking of Multimedia Contents III, P. W. Wong and E. J. Delp, Eds., vol. 4314 of Proceedings of SPIE, San Jose, Calif, USA, January 2001.

[21] W. M. Lawton, "Necessary and sufficient conditions for constructing orthonormal wavelet bases," Journal of Mathematical Physics, vol. 32, no. 1, pp. 57-61, 1991.

[22] J. M. Hereford and D. W. Roach, "Image compression using parameterized wavelets with feedback," in Independent Component Analyses, Wavelets, and Neural Networks, vol. 5102 of Proceedings of SPIE, pp. 267-277, Orlando, Fla, USA, April 2003.

[23] G. Regensburger and O. Scherzer, "Symbolic computation for moments and filter coefficients of scaling functions," Annals of Combinatorics, vol. 9, no. 2, pp. 223-243, 2005.

[24] M. J. Lai and D. Roach, "Parameterizations of univariate orthogonal wavelets with short support," in Approximation Theory X: Wavelets, Splines, and Applications, J. Stoeckler, C. K. Chui, and L. L. Schumaker, Eds., pp. 369-384, Vanderbilt University Press, Nashville, Tenn, USA, 2002.

[25] J. P. Li and Y. Y. Tang, "General analytic construction for wavelet lowpassed filters," in Proceedings of the 2nd International Conference on Wavelet Analysis and its Applications (WAA '01), pp. 314-320, Hong Kong, China, December 2001.

[26] L. Shen, H. H. Tan, and J. Y. Tham, "Symmetric-antisymmetric orthonormal multiwavelets and related scalar wavelets," Applied and Computational Harmonic Analysis, vol. 8, no. 3, pp. 258-279, 2000. 


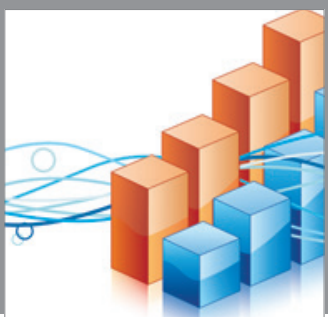

Advances in

Operations Research

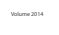

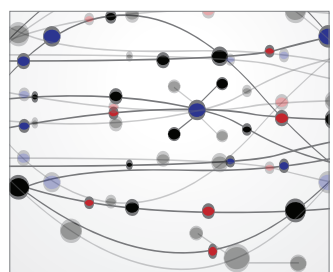

\section{The Scientific} World Journal
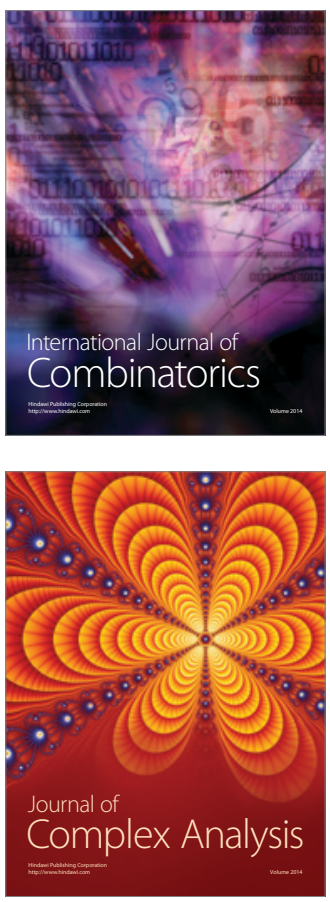

International Journal of

Mathematics and

Mathematical

Sciences
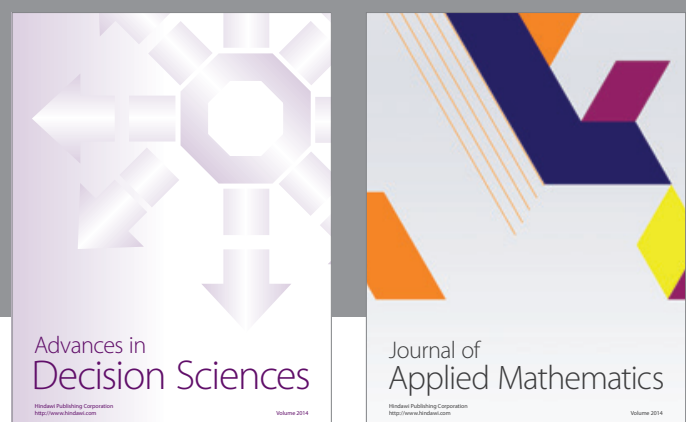

Journal of

Applied Mathematics
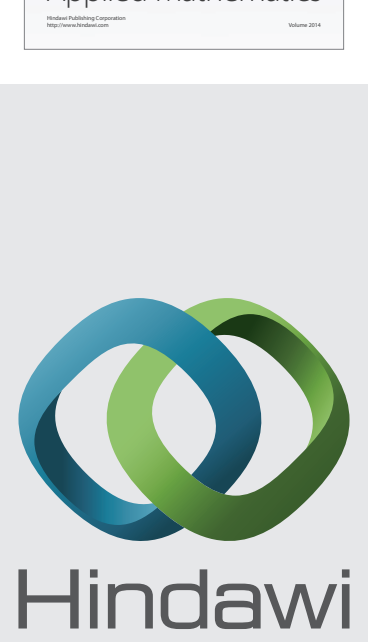

Submit your manuscripts at http://www.hindawi.com
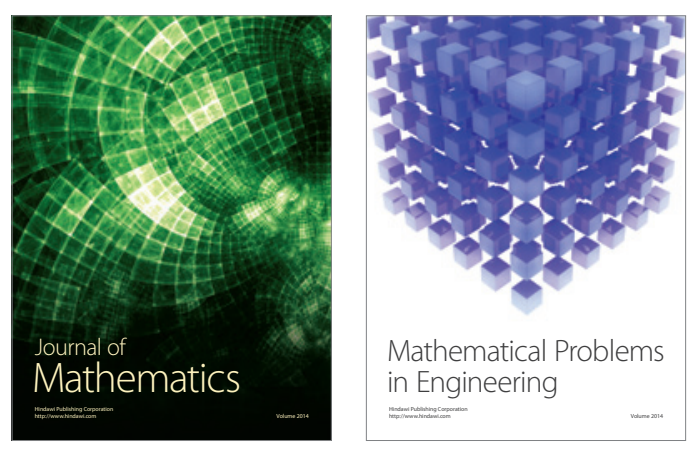

Mathematical Problems in Engineering
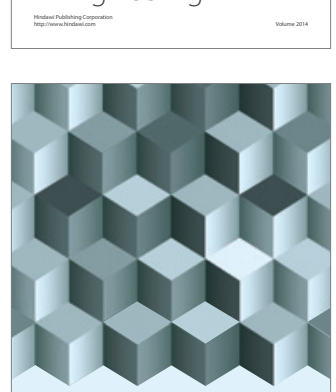

Journal of

Function Spaces
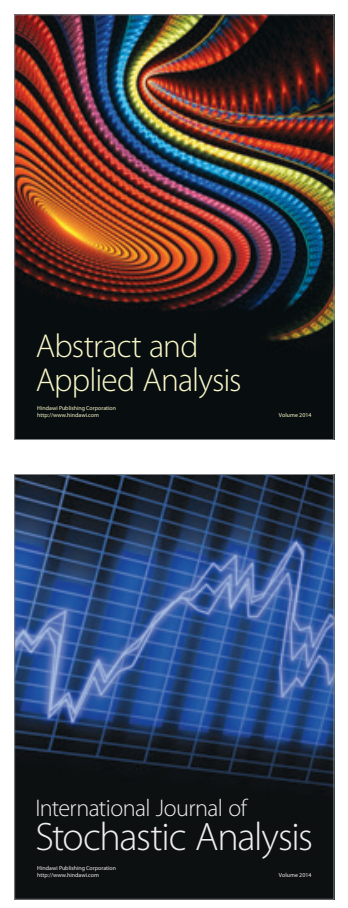

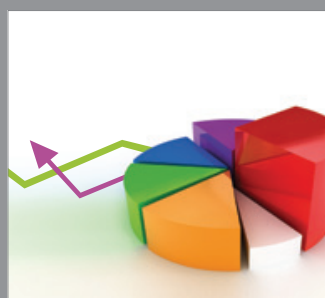

ournal of

Probability and Statistics

Promensencen
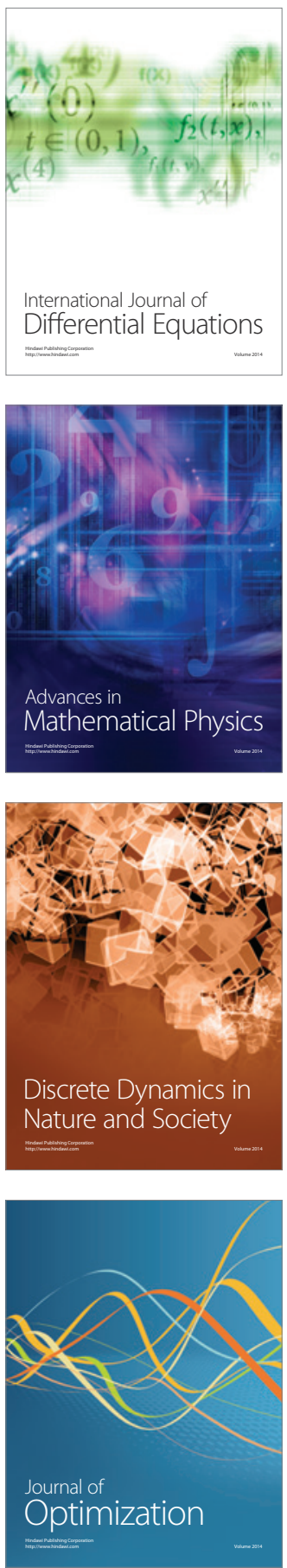